\title{
Fear of childbirth in urban and rural regions of Turkey: Comparison of two resident populations
}

\author{
Filiz Okumus, ${ }^{1}$ Nevin Sahin ${ }^{2}$ \\ ${ }^{1}$ Department of Midwifery, Istanbul Medipol University, College Of Health Sciences, Istanbul, Turkey \\ ${ }^{2}$ Department of Nursing, Istanbul University Florence Nightingale Faculty of Nursing, Women Health and Disease Nursing, Istanbul, Turkey
}

\begin{abstract}
OBJECTIVE: Childbirth is a natural physiological event experienced by many women; however, it is frequently also a source of fear in women. Rates of cesarean sections in Turkey are higher in the urban areas than in the rural areas. We hypothesized that lower fear of childbirth (FOC) rates would be observed in the city having the lowest cesarean section rates in Turkey. This study aimed to compare FOC in women in two resident populations: one in a rural area and the other in an urban area.
\end{abstract}

METHODS: This study was conducted on 253 pregnant women in Istanbul, a large urban municipality, and Siirt, a city in rural Turkey. A descriptive information form and the A version of the Wijma Delivery Expectancy/Experience Questionnaire (W-DEQ) were used.

RESULTS: Severe FOC levels were recorded in women in the Istanbul sample; moreover, these levels were higher than those recorded in women in the Siirt sample. In addition, women in the Istanbul sample preferred vaginal birth to cesarean section and had greater FOC, a finding which demonstrates that women prefer vaginal birth even though they have a higher FOC level and live in a city with high cesarean section rates. Where women live (rural versus urban areas) affects their perception of birth and consequently, their FOC levels.

CONCLUSION: The results of this study suggest that further cross-cultural and regional research is needed for better understanding FOC and factors associated with elevated FOC levels within each cultural setting.

Keywords: Fear of childbirth, WDEQ-A, Pregnancy.

$\mathrm{C}$ hildbirth, a normal physiological event, is one of the most fulfilling and powerful experiences in a woman's life. Throughout pregnancy and during childbirth, women face many challenges. The fear of childbirth (FOC), which may be one of those challenges, can be an important source of distress for pregnant women and their caregivers.
Although not an isolated occurrence, FOC is associated with several problems, including anxiety, low self-esteem, depression, dissatisfaction with the partnership, and lack of support [1]. It also affects women leading to an avoidance of pregnancy, maternal and fetal stress, and an increase in maternal requests for cesarean sections. The literature

Received: May 24, 2017 Accepted: July 17, 2017 Online: October 20, 2017

Correspondence: Dr. Filiz OKUMUS. Istanbul Medipol Universitesi Halic Yerleskesi, Ataturk Bulvarı, Fatih, Istanbul, Turkey Tel: +9021245348 88 e-mail: filizokumush@gmail.com

(c) Copyright 2017 by Istanbul Northern Anatolian Association of Public Hospitals - Available online at www.kuzeyklinikleri.com 
indicates that psychological problems during pregnancy are related to negative perinatal outcomes, such as preterm birth, low birth weight, and newborn anomalies [2]. A study conducted on 2.206 pregnant women in Norway reported that FOC extended the duration of the birth process for as much as an hour and a half [3]. In another study on Swedish women, FOC caused an increase in the use of vacuum extractors [4].

Women's labor and birth mode have been affected by FOC at both individual and population levels. Maternal requests for cesarean sections have been persistently long-documented as a cause for the procedure's increasing rates in recent decades. It has also been documented that often, FOC is an underlying factor in maternal requests for cesarean sections. Therefore, FOC is considered to be the main reason for the increase in cesarean section rates [5].

Despite a lack of scientific evidence indicating substantial maternal and perinatal benefits of an increased number of cesarean sections, the rates of this procedure continue to increase worldwide (Italy, 38\%; Colombia, 43\%; Mexico, 46\%; Iran, 48\%; Egypt, 52\%; and Brazil, 56\%) [6]. In the United States, cesarean section rates increased for the 12 th consecutive year, to $32.8 \%$ in 2012 , which is a proportional increase of $56 \%$ since 1996 [7]. The World Health Organization (WHO) recommends that to optimize maternal and neonatal outcomes, cesarean section rates should not exceed $10-15$ per 100 live births [8]. The Statement on Cesarean Section Rates recently released by WHO, which summarizes the results of systematic reviews and analyses conducted, indicate that cesarean section rates higher than $30 \%$ are not associated with reductions in maternal and newborn mortality rates [8].

Cesarean section rates in Turkey during 20022014 increased by approximately 2.5-fold (from $21 \%$ to $51 \%$ ) [9]. Cesarean rates in Istanbul and Siirt were $57 \%$ and $22 \%$, respectively; the latter demonstrated the lowest cesarean rate in Turkey [9-10]. In addition, these rates were $69.5 \%$ in private hospitals and $35.5 \%$ in state hospitals in Turkey. Because cesarean section rates are on the rise in different hospitals, cities, and even in regions of Turkey, FOC might be a potential factor in this increase. Although the impact of FOC in some countries has been documented in the literature, a limited number of studies have focused on FOC in Turkey [11]. In light of the statistical evidence described in the preceding paragraphs, we aimed to determine whether there are interregional differences in terms of FOC. We hypothesized that lower FOC rates would be observed in the city having the lowest cesarean section rate in Turkey.

\section{MATERIALS AND METHODS}

\section{Setting}

This cross-sectional study was conducted between January and February 2013 with pregnant women who were admitted to health centers in Istanbul (a large urban municipality, the Istanbul sample) and Siirt (a city in rural Turkey, the Siirt sample). The health center in the city of Siirt is a state hospital which is the site of approximately 2.400 births per year (with a cesarean section rate of $24 \%$ ). The health center in Istanbul is a private hospital in which approximately 2.500 births occur per year (with a cesarean section rate of $60 \%$ ).

\section{Participants and recruitment}

Eligible participants were recruited from obstetric outpatient clinics of hospitals in Siirt and Istanbul. The study samples included pregnant women who could speak Turkish, aged $18-45$ years, and in the second trimester of pregnancy. Women with pregnancy complications (e.g., placenta previa, oligohydramnios, pre-eclampsia, and fetal malformation) and chronic illnesses (e.g., cardiac diseases, diabetes mellitus, epilepsy, and psychiatric disorders) were excluded from the study. Of the 155 eligible women in the Istanbul sample, 148 women (95\%) were recruited in the study. Of the 110 eligible women in the Siirt sample, 105 women (95\%) were recruited.

A simple random sampling was used. The participants were randomly selected from eligible women who, during a routine ultrasound screen- 
ing examination, volunteered to take part in the study. The study group consisted of 148 and 105 pregnant women from Istanbul and Siirt, respectively.

\section{Questionnaire}

We designed a 20-item questionnaire that was based on a literature review and included questions related to socio-demographic (i.e., age, education level, type of family, and employment) and obstetric (i.e., age at first birth, gravidity, parity, plan of pregnancy, previous birth(s), and preferred childbirth mode) data. Childbirth-related fear was assessed using the A version of the Wijma Delivery Expectancy/Experience Questionnaire (W-DEQA). The participants' socio-demographic and obstetric data were examined in terms of their effects on FOC.

In 1998, W-DEQ Version A was developed by Klass and Barbro Wijma in Sweden; its Turkish adaptation was completed by Korukcu et al. [12] W-DEQ-A is a prepartum version of the scale and comprises 33 items that assess the FOC level based on women's cognitive appraisals and expectations about delivery. The responses were scored from 0 to 5 and were measured using a 6 -item Likerttype scale; total scores ranged from 0 to 165 . The cut-off level was 85 : scores of $\leq 37$ indicate a mild fear level, scores of 38-65 indicate a moderate fear level, scores of 66-84 indicate a severe fear level, and scores of $\geq 85$ demonstrate clinical fear. Using Chronbach's a coefficient of 0.89 [12], the Turkish version of the W-DEQ-A was found to be reliable and valid. In the present study, Chronbach's a was determined to be 0.89 .

\section{Ethical considerations}

Ethical approval for the study was obtained from the local ethical committee of the Istanbul Medipol University (protocol number: 2013/1084009816). The study participants received verbal information about the study, were given the opportunity to ask questions, and provided written informed consent forms. The questionnaire was completed using a face-to-face interview technique.

\section{Data analysis}

The distribution of continuous variables, such as age, age at first birth, and W-DEQ total score, were evaluated using the Shapiro-Wilk test. Normally and non-normally distributed variables are shown as mean \pm standard deviation (mean $\pm \mathrm{SD}$ ) and median (min-max), respectively. W-DEQ total scores are expressed by both mean \pm SD and median $(\min -\max )$.

Either Mann-Whitney U test or the KruskalWallis test was used for comparing non-normally distributed variables. Age was compared via the Student's t-test. Categorical variables were evaluated using chi-square tests. The Mann-Whitney $\mathrm{U}$ test with the Bonferroni correction and a $Z_{\text {-test }}$ with the Bonferroni correction were performed post-hoc for the Kruskal-Wallis and chi-square tests, respectively. A p value of $<0.05$ was accepted as statistically significant. All statistical analyses were performed using IBM SPSS Statistics 21.0 (IBM Corp. Released 2012. IBM SPSS Statistics for Windows, Version 21.0. Armonk, NYः IBM Corp).

\section{RESULTS}

\section{Sample characteristics}

Characteristics of the two samples are presented in Table 1. The socio-demographic characteristics of both samples were well-matched, with significant differences $(p<0.05)$ in age, education levels, type of family, and employment. Participants in the Istanbul sample were more likely to have higher education and employment levels and nuclear family characteristics than those in the Siirt sample.

Age at the time of the first pregnancy was 28 and 20 in the Istanbul and Siirt samples, respectively. Women in Siirt were more likely to become pregnant during their teenage years than those in Istanbul (49.5\% and $2.0 \%$, respectively). The mean number of pregnancies was $1.7 \pm 1.1$ ( $\min : 1$; $\max : 8$ ) and $3.6 \pm 2.2$ (min: $1 ; \max : 10$ ) for the Istanbul and Siirt samples, respectively.

While the ratio of primigravid women (57.4\%) was higher in the Istanbul sample, the ratio of multigravid women $(81.9 \%)$ was higher in the Siirt sam- 
ple. The mean number of births was $0.5 \pm 0.7$ ( $\min : 0$; max: 3 ) and 2.4 \pm 2.1 (min: 0; max: 9) for the Istanbul and Siirt samples, respectively. More than half of the women in the Istanbul sample were nulliparous $(64.9 \%)$, and more than half of the women in the $\mathrm{Si}$ irt sample were multiparous (60\%). The previous cesarean section rates performed on participants in the Istanbul sample was significantly greater than that performed on those in the Siirt sample (Table 1).

In the Siirt sample, the percentage of women who preferred the vaginal birth mode $(p<0.001)$ was higher than that who preferred the cesarean section mode $(\mathrm{p}<0.001)$. In the Istanbul sample, 87\% women who had previously undergone cesarean sections expressed a preference for cesarean section as the mode for their next childbirth. This percentage was just $13 \%$ in the Siirt sample.

\section{FOC level}

The mean WDEQ-A score was $97.3 \pm 19.8$ (median: $96.5 ;$ min: 28; max: 150) in the Istanbul sample and 77.7 \pm 16.2 (median: 75.0; min: 34; max:135) in the Siirt sample. Women in the Istanbul sample had higher WDEQ-A scores that those in the Siirt sample $(p<0.05)$. For each sample, WDEQ-A scores were compared across a range of socio-demographic and obstetric factors to identify the groups of women with higher FOC levels (Tables 2 and 3).

A box plot of these scores (Fig. 1) indicates that the cut-off point of 85 clearly distinguishes these two groups of women. Participant residents of Istanbul were observed to have a clinical level of $75.7 \%$ FOC, while the Siirt participants had an FOC severity level of $60 \%$.

\section{The Istanbul sample}

There were no differences with respect to age, age at first pregnancy, education level, type of family, employment, gravidity, planned pregnancy, and previous birth mode among the WDEQ-A scores of participants in the Istanbul sample. Also in the Istanbul sample, significantly higher WDEQ-A scores were identified for nulliparous women. Moreover, being nulliparous was associated with a higher FOC level in this group. Although there were significant differences $(p<0.05)$ among nulliparous, primiparous, and multiparous women, there were no significant differences in their pairwise comparisons ( $p>0.05)$ (Table 2).

A preference for the vaginal birth mode was found to be associated with greater WDEQ-A scores. Eighty-three participants $(56.1 \%)$ indicated that they would prefer a vaginal birth mode. These women recorded WDEQ-A scores that were substantially higher (median: 100) than those of women who preferred a cesarean section mode (median: 89) $(\mathrm{p}<0.05)($ Table 2).

\section{The Siirt sample}

WDEQ-A scores of women in the Siirt sample demonstrated no differences with respect to age at first pregnancy, education level, employment, gravidity, parity, planned pregnancy, previous childbirth method, and preferred childbirth method. In the Siirt sample, 51 women were in their teen years at the time of their pregnancy. Significantly higher WDEQ-A scores were identified for age at first birth in women aged 25-29 years. The nuclear family characteristic was also found to be associated with greater WDEQ-A scores (Table 3).

\section{DISCUSSION}

Numerous studies conducted in Scandinavian countries have suggested that a substantial num-

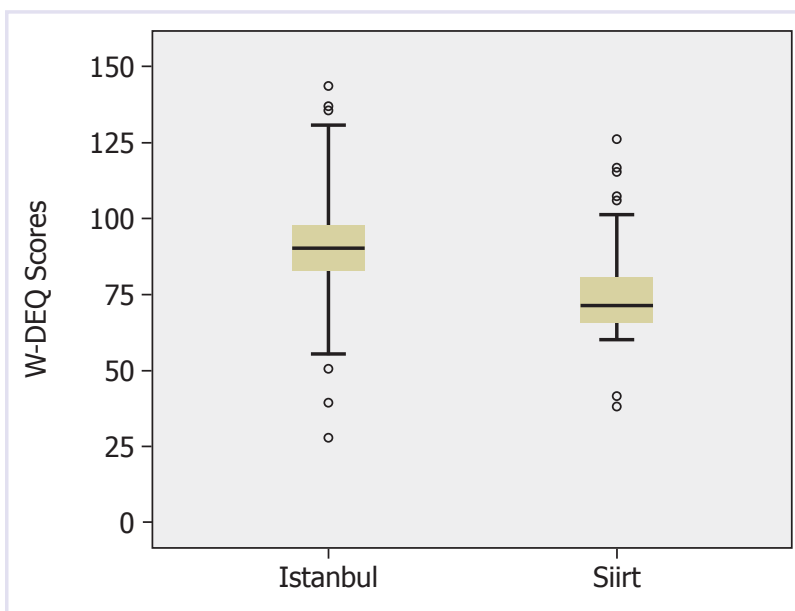

FIGURE1. Distribution of W-DEQ scores among the groups 
TABLE 1. Characteristics of the Istanbul and Siirt samples $(n=253)$

\begin{tabular}{|c|c|c|c|}
\hline & $\begin{array}{c}\text { Istanbul } \\
\mathrm{n}=148 \\
\mathrm{n}(\%)\end{array}$ & $\begin{array}{c}\text { Siirt } \\
n=105 \\
n(\%)\end{array}$ & $\mathrm{p}$ \\
\hline \multicolumn{4}{|l|}{ Age (years) } \\
\hline Mean \pm SD & $30.3 \pm 4.1$ & $27.1 \pm 5.6$ & $<0.001$ \\
\hline (Min-Max) & $(21-42)$ & $(14-41)$ & \\
\hline \multicolumn{4}{|l|}{ Education } \\
\hline At most elementary & $6(4.1)$ & $75(71.4)$ & $<0.001$ \\
\hline More than elementary & $142(95.9)$ & $30(28.6)$ & \\
\hline \multicolumn{4}{|l|}{ Type of family } \\
\hline Nuclear & $141(95.3)$ & $41(39.0)$ & $<0.001$ \\
\hline Extended & $7(4.7)$ & $64(61.0)$ & \\
\hline \multicolumn{4}{|l|}{ Employment } \\
\hline Yes & $94(63.5)$ & $7(6.7)$ & $<0.001$ \\
\hline No & $54(36.5)$ & $98(93.3)$ & \\
\hline \multicolumn{4}{|l|}{ Age at first pregnancy } \\
\hline Median & 28.0 & 20.0 & $<0.001$ \\
\hline (Min-Max) & $(18-38)$ & $(14-41)$ & \\
\hline \multicolumn{4}{|l|}{ Gravidity } \\
\hline Primigravid & $85(57.4)$ & $19(18.1)$ & $<0.001$ \\
\hline Multigravid & $63(42.6)$ & $86(81.9)$ & \\
\hline \multicolumn{4}{|l|}{ Parity } \\
\hline Nulliparous & $96(64.9)^{a}$ & $23(21.9)^{a}$ & $<0.001$ \\
\hline Primiparous & $38(25.7)$ & $19(18.1)$ & \\
\hline Multiparous & $14(9.5)^{\mathrm{b}}$ & $63(60.0) 0^{b}$ & \\
\hline \multicolumn{4}{|l|}{ Plan of pregnancy } \\
\hline Planned & $125(84.5)$ & $79(75.2)$ & 0.095 \\
\hline Unplanned & $23(15.5)$ & $26(24.8)$ & \\
\hline \multicolumn{4}{|l|}{ Preferred mode of birth } \\
\hline Vaginal & $83(56.1)$ & $97(92.4)^{a}$ & $<0.001$ \\
\hline Cesarean & $45(30.4)^{b}$ & $7(6.7)$ & \\
\hline Uncertain & $20(13.5)^{c}$ & $1(1.0)$ & \\
\hline \multirow{2}{*}{\multicolumn{4}{|c|}{$\begin{array}{l}\text { Previous mode of birth } \\
\text { (Multiparous only) }(n=134)\end{array}$}} \\
\hline & & & \\
\hline Vaginal & $18(34.6)$ & $78(95.1)$ & $<0.001$ \\
\hline Caesarean section & $34(65.4)$ & $4(4.9)$ & \\
\hline
\end{tabular}

$a, b, c$ Indicates statistically significant difference $(p<0.05)$ in pairwise comparison of the subgroups.

ber of women experience high FOC levels [13-14]. However, a limited number of non-Scandinavian studies on the prevalence of FOC have been documented [15]. Some cross-cultural studies have also been reported explaining factors affecting FOC [15-16]. However, a limited number of studies have focused on FOC in Turkey [11].

Unlike previously published research, the pres- 
TABLE 2. Istanbul

Mean \pm SD

Median

(Min-Max)

Age (years)

$\leq 24$

25-29

30-34

$\geq 35$

Age at first pregnancy

$\leq 19$

20-24

25-29

$\geq 30$

Education

Elementary \& Secondary

High School

University

Type of family

Nuclear

Extended

Employment

Yes

No

Gravidity

Primigravid

Multigravid

Parity

Nulliparous

Primiparous

Multiparous

Preferred mode of birth

Vaginal

Caesarean section

Uncertain

Plan of pregnancy

Planned

Unplanned

Previous mode of birth

Vaginal

Caesarean section
$100.29 \pm 20.57$

$99.39 \pm 16.85$

$96.72 \pm 24.38$

$92.48 \pm 13.27$

$95.33 \pm 3.51$

$98.36 \pm 15.69$

$98.59 \pm 18.82$

$94.80 \pm 23.86$

$103.88 \pm 9.40$

$96.29 \pm 16.97$

$97.24 \pm 21.49$

$97.32 \pm 20.23$

$97.57 \pm 7.64$

$97.66 \pm 20.73$

$96.76 \pm 19.12$

$98.01 \pm 19.06$

$96.41 \pm 20.87$

63

96

38

14

83

45

20

125

23

18

34

$92.66 \pm 21.04$

$91.07 \pm 10.23$

$102.86 \pm 16.61$

$89.71 \pm 24.34$

$91.55 \pm 12.84$

$98.14 \pm 19.30$

$92.91 \pm 22.23$

$96.67 \pm 14.54$

$89.88 \pm 20.32$
93.0 (81-143)

0.466

$100.0(59-145)$

$94.0(28-150)$

$92.0(54-117)$

$95.0(92-99)$

0.942

$93.0(77-143)$

$97.0(59-145)$

$97.0(28-150)$

$105.0(90-117)$

97.0 (59-143)

$95.5(28-150)$

$97.0(28-150)$

0.832

96.0 (90-107)

94.5 (39-150)

$99.0(28-130)$

99.0 (39-145)

0.262

$93.0(28-150)$

$100.5(39-150)$

0.017

90.0 (28-144)

$91.0(77-117)$

$100.0(65-150)^{\mathrm{a}, \mathrm{b}}$

$<0.001$

$89.0(28-134)^{\mathrm{a}}$

$88.0(74-128)^{\mathrm{b}}$

0.679
99.0 (39-150)

0.287

$93.0(28-130)$

96.0 (76-124)

88.5 (28-144) $a, b$ Indicates statistically significant difference $(p<0.05)$ in pairwise comparison of the subgroups. ent study was conducted on women having different cultural and socio-demographic properties. In addition, the study was conducted to fill the gap in knowledge with respect to specific aspects of FOC in Turkish women. As is true in many societies worldwide, the Turkish health authority strongly 
TABLE 3. Siirt

n

Mean \pm SD

Median

(Min-Max)

Age (years)

$\leq 24$

25-29

30-34

$\geq 35$

Age at first pregnancy

$\leq 19$

20-24

25-29

Not attended a school

Education

Illiterate

Elementary

Secondary

High school \& University

Type of family

Nuclear

Extended

Employment

Yes

No

Gravidity

Primigravid

Multigravid

Parity

Nulliparous 23

Primiparous 19

Multiparous 63

Preferred mode of birth

Vaginal

Caesarean section

Plan of pregnancy

Planned

Unplanned

Previous mode birth

Vaginal

Caesarean section

32

37

27

9

51

39

13

36

36

39

20

10

41

64

7

98

19

86

$23 \quad 81.00 \pm 20.92$

$83.11 \pm 13.85$

97

7

79

26

78

4
$74.90 \pm 14.48$

$77.59 \pm 16.70$

$80.89 \pm 17.13$

$74.07 \pm 15.13$

$76.11 \pm 13.56$

$75.69 \pm 15.64$

$76.92 \pm 16.24$

$87.38 \pm 16.95$

$75.03 \pm 14.05$

$75.03 \pm 14.05$

$77.03 \pm 14.28$

$78.10 \pm 20.63$

$89.40 \pm 18.26$

$81.46 \pm 20.26$

$75.33 \pm 12.62$

$92.29 \pm 28.57$

$76.68 \pm 14.68$

$81.74 \pm 22.73$

$76.84 \pm 14.44$

$78.31 \pm 16.00$

$71.57 \pm 19.67$

$78.27 \pm 17.96$

$76.08 \pm 9.21$

$77.10 \pm 14.34$

$71.00 \pm 22.14$
$74.0(51-122)$

0.782

74.0 (53-132)

75.0 (34-111)

78.0 (56-102)

$72.0(51-132)^{\mathrm{a}}$

75.0 (34-122)

$81.0(64-113)^{\mathrm{a}}$

$77.0(34-111)$

0.201

77.0 (34-111)

0.201

74.0 (51-132)

68.5 (53-122)

86.5 (69-113)

$81.0(34-132)$

0.037

73.0 (51-111)

$106.0(39-113)$

0.061

74.0 (34-132)

76.0 (51-122)

0.927

74.5 (34-132)

76.0 (51-122)

0.113

82.0 (64-119)

74.0 (34-132)

75.0 (34-132)

77.0 (39-94)

0.599

74.0 (34-132)

0.935

77.5 (56-96)

74.0 (34-132)

77.5 (39-90)

aindicates statistically significant difference $(p<0.05)$ in pairwise comparison of the subgroups.

promotes the vaginal birth mode. However, there are significant differences between western and eastern Turkey in its perform. Siirt and Van, two cities located in the eastern Anatolian region of Turkey have the lowest cesarean section rates (22\%) [10]. We opted to include participants from Siirt 
for examining correlations between FOC and the preferred method of childbirth.

Women the Istanbul sample were older and had higher educational levels than those in the Siirt sample. The rates of those living in nuclear families, working, and whose income exceeded expenses were higher for women in the Istanbul sample. Data from the Turkey Demographic and Health Survey [17] show that eastern and western Turkey are not equal in terms of education levels. The findings of this study related to the socio-demographic characteristics of pregnant women were consistent with Turkey Demographic and Health Survey data [17]. An analysis of the Siirt sample also revealed that participants with extended families had a significantly lower FOC than those in nuclear families. This result is in line with the literature [18] and indicates that women who receive support from close relatives may have reduced FOC.

Studies have observed that primiparous women report higher FOC levels during their pregnancies [14]. We found no significant differences in FOC between primigravid and multigravid women in either sample. This result is consistent with findings reported by Nilsson et al. [18] and Ternström et al. [19]. Although there were significant differences among nulliparous, primiparous, and multiparous women, there were no significant differences in their pairwise comparisons. This finding is not consistent with previously published results [13] and may result for numerous reasons, such as social, cultural, ethnical, socio-demographic, or even religious factors.

Women live in a social environment; they communicate with other women who have experienced vaginal childbirth and/or cesarean sections. Sen et al. [20] reported a correlation between communication about delivery with other parous women and attitudes related to fear of delivery. The authors also noted, however, that it was not clear whether or not the prevalence of shared positive birth stories contributed to the childbirth experience. Some pregnant women (6-10\% of the participants) reported to have experienced anxiety and anxiety-related symptoms/or FOC in their daily lives [21]. A lack of preparation (for several reasons) for childbirth and a lack of sufficient information about birth and pregnancy were found to be related to women's FOC [22]. Nulliparous women tend to be more anxious about the childbirth process because it represents a situation that is unknown to them [23]. This kind anxiety and/or fear may be attributed to a complexity of emotions during pregnancy [24]. Future research should focus on these emotional factors in an attempt to understand which of them contribute to FOC and in what ways. We suggest that women with or without FOC should be closely monitored throughout their first or/and subsequent pregnancies.

Previous studies have shown that women who have experienced a cesarean section are more likely to opt for a subsequent operative birth $[25,26]$. Our results were also in line with those of previous studies. Of the women in the Istanbul sample who have undergone a cesarean section, $87 \%$ preferred cesarean section as the mode of delivery in their subsequent birth(s). In a study on western Australian women, Fenwick et al. [27] reported that discouragement by clinicians to choose a vaginal birth after having a cesarean section plays a role in the increased rate of cesarean sections. It has also been acknowledged that women are more likely to request a repeat cesarean than a primary cesarean because of the powerful influence of their clinicians [28]. The influence of clinicians was confirmed in our study. We also believe that midwifery support and antenatal counseling could have a positive effect on the decrement of cesarean section rates.

It is interesting to note that we found regional differences in the participants' preferences for the birth mode. Women in the Istanbul sample (30.4\%) were more likely to prefer a cesarean section than their Siirt counterparts (6.7\%). This pattern was not reflected in the actual cesarean section rates reported for each of the cities. During the period of the study (2013), the cesarean section rates were $57 \%$ in Istanbul and $22 \%$ in Siirt $[9,10]$. This difference may be attributed to the socio-economic status because we recruited our Istanbul sample from a private hospital. However, participants in our Siirt sample had been admitted to a state hospital. The present study revealed that women in the Istanbul 
sample experienced higher FOC levels than those in the Siirt sample. FOC demonstrated by participants in the Istanbul sample was a more commonly observed phenomenon in women who preferred the vaginal birth mode. Moreover, those in the Istanbul sample who preferred the vaginal birth mode had significantly higher FOC scores.

It is interesting to note that women in the Siirt sample who preferred the vaginal birth mode had no significantly different ( $p>0.05$ ) FOC levels compared with those who preferred the cesarean section mode. This finding may be attributed to childbirth, which is, itself, a factor in FOC. Therefore, childbirth may also be regarded to as a woman's fate, which must be experienced.

Women in the Istanbul sample demonstrated significantly higher preference rates $(p<0.05)$ for the cesarean section mode than those in the Siirt sample (30.4\% vs. 6.7\%). However, women in the Istanbul sample who preferred the vaginal birth mode had significantly higher FOC scores than those who preferred the cesarean section mode. The vaginal birth-related fear scores were significantly higher $(p<0.05)$ in women the Istanbul sample than in those in the Siirt sample. Moreover, the clinical fear score in the Istanbul sample $(75.7 \%$ of all women) was significantly higher $(p<0.05)$ tahn that in the Siirt sample (23.8\% of all women). We think that it is difficult to explain the reason(s) for FOC because it is the result of multiple factors. We also believe that future studies should be conducted for clarifying the association between FOC and factors related to childbirth.

Severe FOC levels were recorded for participants in the Siirt sample; however, these FOC levels were lower than those recorded for participants in the Istanbul sample. In addition, women in the Istanbul sample who preferred the vaginal birth mode to the cesarean section mode had higher FOC scores. This finding indicates that women prefer vaginal birth even if they have higher FOC levels and live in a city with a high rate of cesarean sections. Where women live (a rural area versus an urban area) affects their perception of birth and consequently, their FOC. To reduce the cesarean section rates, which are increasing worldwide, it will be very im- portant to analyze the experiences of women who live in rural areas. The results of this study suggest that further cross-cultural and regional research is needed to better understand FOC as well as factors associated with elevated fear levels within each cultural setting. In addition, we suggest that a governmental midwifery-led care model be implemented within the health systems for reducing negative perceptions of childbirth, handling fear associated with it as well as extending support to all Turkish women who experience childbirth.

\section{Limitations}

Limitations of the current study must be noted. The samples used in the present study were relatively small, which may have impacted the power of the study. Istanbul and Siirt are extremely different cities with respect to several aspects. Istanbul ranks as the world's seventh largest city based on population and is the largest city in Europe. The population of the Istanbul Metropolitan $\mathrm{Mu}$ nicipality, reported to be 14.377 .019 at the end of 2014 , comprises $19 \%$ of 'Turkey's population. The remarkable growth is, and continues to be, largely fueled by immigrants from eastern Turkey who seek employment and improved living conditions [29]. In contrast, Siirt is a city with one of the lowest populations and the highest fertility rate in the southeastern Anatolia region. Turkish Statistical Institute data indicate that the total fertility rate in 2014 was 3.86 in Siirt and 1.77 in Istanbul [30]. In addition, cesarean section rates in Istanbul and Siirt were $57 \%$ and $22 \%$, respectively $[9,10]$. These statistics prompted us to explore cesarean section rates in Turkey and to determine some clue for interregional differences.

Conflict of Interest: No conflict of interest was declared by the authors.

Financial Disclosure: The authors declared that this study has received no financial support.

Authorship contributions: Concept - F.O.; Design - F.O.; Supervision - F.O.; Materials - N.S, F.O.; Data collection \&/or processing - F.O.; Analysis and/or interpretation - F.O.; Literature search - F.O., N.S.; Writing - F.O; Critical review - F.O. 


\section{REFERENCES}

1. Saisto T, Halmesmaki E. Fear of childbirth: a neglected dilemma. Acta Obstet Gynecol Scand 2003;82:201-8. [CrossRef]

2. Zelkowitz P, Papageorgiou A. Easing maternal anxiety: an update. Womens Health (Lond Engl) 2012;8:205-13. [CrossRef]

3. Adams SS, Eberhard-Gran M, Eskild A. Fear of childbirth and duration of labour: a study of 2206 women with intended vaginal delivery. BJOG 2012;119:1238-46. [CrossRef]

4. Sydsjö G, Sydsjö A, Gunnervik C, Bladh M, Josefsson A. Obstetric outcome for women who received individualized treatment for fear of childbirth during pregnancy. Acta Obstet Gynecol Scand 2012;91:44-9. [CrossRef]

5. Hildingsson I. Swedish couples' attitudes towards birth, childbirth fear and birth preferences and relation to mode of birth - a longitudinal cohort study. Sex Reprod Healthc 2014;5:75-80. [CrossRef]

6. WHO. World Health Statistics 2015. Available at: http://apps. who.int/iris/bitstream/10665/170250/1/9789240694439_eng. pdf. Accessed Nov, 2016.

7. Martin JA, Hamilton BE, Osterman MJ, Curtin SC, Matthews TJ. Births: final data for 2012. Natl Vital Stat Rep 2013;62:1-68.

8. World Health Organization. WHO statement on caesarean section rates. http://apps.who.int/iris/bitstream/10665/161442/1/WHO_RHR_15.02_eng.pdf?ua=1 (accessed in November 11, 2016). 2015.

9. Ministry of Health of Turkey. The 2014 Ministry of Health of Turkey Health Statistics Yearbook. 2014:http://ekutuphane. sagem.gov.tr/kitaplar/saglik_istatistikleri_yilligi_2014.pdf (accessed in November 2016, 2016).

10. Ministry of Health of Turkey. 2014 Statistics of Public Hospital of Turkey. 2014:http://rapor.saglik.gov.tr/tkhk2014/tkhk2014. pdf (Accessed in November 2016, 2016).

11. Sercekus P, Okumus H. Fears associated with childbirth among nulliparous women in Turkey. Midwifery 2009;25:155-62.

12. Korukcu O, Kukulu K, Firat MZ. The reliability and validity of the Turkish version of the Wijma Delivery Expectancy/Experience Questionnaire (W-DEQ) with pregnant women. J Psychiatr Ment Health Nurs 2012;19:193-20. [CrossRef]

13. Fenwick J, Gamble J, Nathan E, Bayes S, Hauck Y. Pre- and postpartum levels of childbirth fear and the relationship to birth outcomes in a cohort of Australian women. J Clin Nurs 2009;18:667-77.

14. Wijma K, Soderquist J, Wijma B. Posttraumatic stress disorder after childbirth: a cross sectional study. J Anxiety Disord 1997;11:587-97. [CrossRef]

15. Haines H, Pallant JF, Karlström A, Hildingsson I. Cross-cultur- al comparison of levels of childbirth-related fear in an Australian and Swedish sample. Midwifery 2011;27:560-7. [CrossRef]

16. Lukasse M, Schei B, Ryding EL. Prevalence and associated factors of fear of childbirth in six European countries. Sex Reprod Healthe 2014;5:99-106. [CrossRef]

17. Hacettepe University Institute of Population Studies. Turkey Demographic and Health Survey 2013. Available at: http:// www.hips.hacettepe.edu.tr/tnsa2013/rapor/TNSA_2013_ ana_rapor.pdf. Accessed Nov, 2016.

18. Nilsson C, Lundgren I, Karlström A, Hildingsson I. Self reported fear of childbirth and its association with women's birth experience and mode of delivery: a longitudinal population-based study. Women Birth 2012;25:114-21. [CrossRef]

19. Ternström E, Hildingsson I, Haines H, Rubertsson C. Higher prevalence of childbirth related fear in foreign born pregnant women-findings from a community sample in Sweden. Midwifery 2015;31:445-50. [CrossRef]

20. Sen E, Alp-Dal N, Dag H, Senveli S. The reasons for delivery: Related fear and associated factors in western Turkey. Nurs pract today 2015;2:25-33.

21. Salomonsson B, Wijma K, Alehagen S. Swedish midwives' perceptions of fear of childbirth. Midwifery 2010;26:327-37. [CrossRef]

22. Saisto T, Salmela-Aro K, Nurmi JE, Halmesmäki E. Psychosocial characteristics of women and their partners fearing vaginal childbirth. BJOG 2001;108:492-8. [CrossRef]

23. Ryding EL, Persson A, Onell C, Kvist L. An evaluation of midwives' counseling of pregnant women in fear of childbirth. Acta Obstet Gynecol Scand 2003;82:10-7. [CrossRef]

24. Hall WA, Hauck YL, Carty EM, Hutton EK, Fenwick J, Stoll $\mathrm{K}$. Childbirth fear, anxiety, fatigue, and sleep deprivation in pregnant women. J Obstet Gynecol Neonatal Nurs 2009;38:567-76.

25. Fenwick J, Staff L, Gamble J, Creedy DK, Bayes S. Why do women request caesarean section in a normal, healthy first pregnancy? Midwifery 2010;26:394-400. [CrossRef]

26. Gamble JA, Creedy DK. Women's preference for a cesarean section: incidence and associated factors. Birth 2001;28:101-10.

27. Fenwick J, Gamble J, Hauck Y. Reframing birth: a consequence of cesarean section. J Adv Nurs 2006;56:121-30. [CrossRef]

28. Haines HM, Rubertsson C, Pallant JF, Hildingsson I. The influence of women's fear, attitudes and beliefs of childbirth on mode and experience of birth. BMC Pregnancy Childbirth 2012;12:55.

29. List of largest cities 2016. Available at: https://en.m.wikipedia. org/wiki/List_of_largest_cities. Accessed Nov 11, 2016.

30. Turkish Statistical Institute 2014. Available at: http://www.turkstat.gov.tr/UstMenu.do?metod=temelist. Accessed Nov 11, 2016. 